\title{
Postoperative delirium
}

\section{A. Rudra, S. Chatterjee*, J. Kirtania, S. Sengupta, G. Moitra, S. Sirohia, R. Wankhade, S. Banerjee}

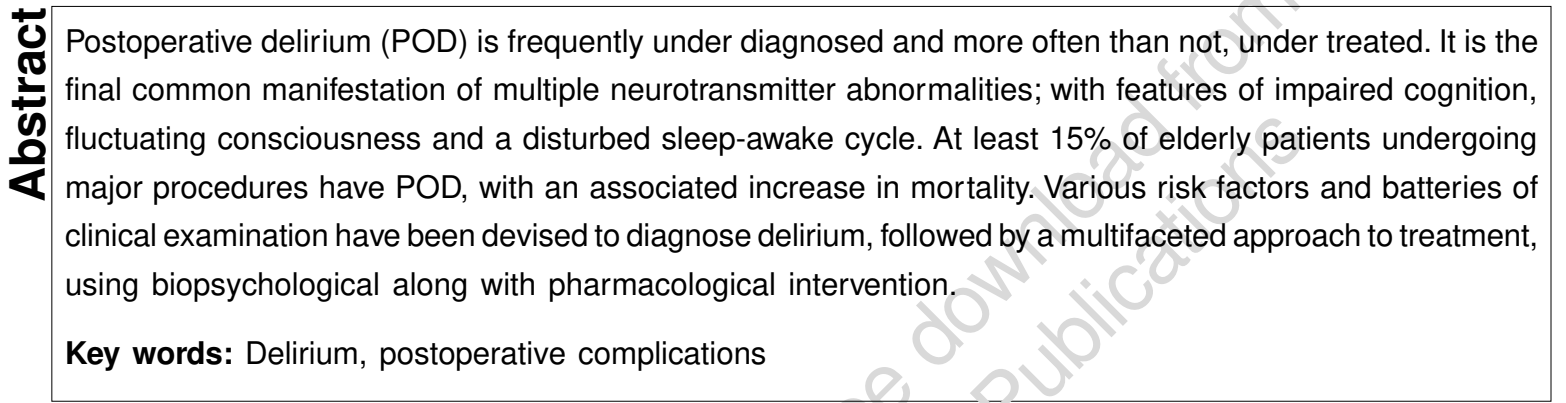

Mental impairment following surgery has been a topic of interest for many years. The acute confusional state known as delirium, - is one of the most frequently encountered complications observed postoperatively. The word delirium is derived from the Latin term meaning "off the track".

Postoperative delirium, a transient mental dysfurwion, can result in increased morbidity, delayed furctional recovery and prolonged hospital stay in the elderly. The distinguishing features of this transient global disorder are impaired cognition, fluctuating level of consciousness, altered psychomotor activity and a disturbed sleep-wake cycle. It is usually seen on the first or second postoperative day and symptoms are often worse at night. The condition can be silent and go unnoticed or it may be misdiagnosed as depression. The postoperative delirium is defined as clinical situations in which patients think and speak incoherently, are disoriented and show impairment of memory and attention. The mini- mental

From:

Apollo Gleneagles Hospital, *Medical College and Hospital, Kolkata, India

Correspondence:

Dr. A. Rudra, 1, Shibnarayan Das Lane, Kolkata - 700 006, India.

E-mail: sumanc_24@yahoo.co.in status examination (MMSE) and other tests can assess speech, consciousness, perception orientation, coherence, memory and motor activity. ${ }^{[1]}$. The MMSE is easy to conduct, reliable and can be used for serial testing in fluctuating conditions. The mortality associated with delirium is high. Delirium is approximately twice the mortality rate as patients without delirium. ${ }^{[2]}$

\section{Incidence and Etiology}

The incidence of delirium is approximately $15 \%$ in elderly patients after major elective procedures but much higher following cardiac procedures and surgery for hip fractures. ${ }^{[3]}$

The mechanism of delirium is controversial. There is probably no final common pathway to delirium, rather delirium is the final common symptom of multiple neurotransmitter abnormalities. One hypothesis for the mechanism behind postoperative delirium is a decrease in the oxidative metabolism of the brain, which results in the decline of neurotransmitter levels within the brain and causes mental dysfunction. Another hypothesis suggests that an increase of serum cortisol from the stress of surgery or anesthesia may be responsible for postoperative confusion. ${ }^{[4,5]}$ 
However another group of workers hypothesized the role of the cholinergic pathways in the pathogenesis of delirium. Production of acetylcholine is decreased in specific medical conditions that precipitate delirium. Serum anticholinergic activity correlates with delirium severity in postoperative patients. Medications with anticholinergic activity frequently can cause confusion in the elderly [Table 1].

\section{Anaesthesia}

Preoperative assessment of the patient's physical and mental status and medications is very important. Preexisting sensory or perceptual deficits compound a patient's chances of developing confusional states. ${ }^{[6]}$ The mainstay of intraoperative preventive measures is maintaining good oxygenation, normal blood pressure, correct drug dosage and normal electrolyte levels. Drug cocktail should be avoided. Atropine, scopolamine and flurazepam should be used only if necessary and the dose should be as low as possible. Glycopyrrolate may be a better choice than atropine as the former is a quarternary amine and should cross the blood-brain barrier less effectively than will atropine. Ambulatory surgery should be encouraged because elderly patients are maintained better in the familiar home environment. Adequate postoperative analgesia, especially in patient who cannot communicate easily because of endotracheal tube or tracheostomy, is crucial. Nurses should be wellversed in detecting the earliest signs of delirium, which in the elderly may be withdrawal rather than agitation. The central nervous depressants, $\mathrm{H}_{2}$-anatagenists, anticholinergies, digitalis, phenytoin, lignocaine and aminophylline should be used with discretion. In general, drugs with short elimination half-lives are preferable to long-acting drugs. ${ }^{[7]}$

\begin{tabular}{ll}
\hline \multicolumn{2}{l}{ Table 1: Drugs commonly associated with delirium } \\
\hline $\begin{array}{l}\text { Class } \\
\text { - Anticholinergic drugs }\end{array}$ & $\begin{array}{l}\text { Examples } \\
\text { Tricyclic antidepressants, } \\
\text { neuroleptics, belladonna alkaloids }\end{array}$ \\
- Opioids & $\begin{array}{l}\text { Morphine, codeine, pethidine } \\
\text { - Benzodiazepines }\end{array}$ \\
- Antiparkinsonian agents & Levodopa/carbidopa, bromocriptine \\
- $\mathrm{H}_{2}$ - receptor blocker & Ranitidine, cimetidine, famotidine. \\
- Cardiovascular agents & $\beta$ - blockers, digoxin, diuretics, \\
& calcium \\
Channel blockers & Cephalosporin, gentamicin \\
- Antibiotics & Phenytoin, carbamazepine \\
- Anticonvulsants & Prednisolone, nonsteroidal agents, \\
- Anti-inflammatory agents & \\
Cyclosporine & Glyburide, glipizide, glimepiride. \\
\hline - Oral - hypoglycemics & \\
\hline
\end{tabular}

\section{Associated Dangers}

Delirium is a common cause of falls, resulting in fractures in old age - and a large proportion of old patients arriving at the emergency department are already delirious on admission. About one third of older patients with hip fractures are usually delirious even before surgery and another third usually develop delirium postoperatively. The longer the waiting time for an operation, the greater the risk that patients will develop delirium. ${ }^{[8]}$ The uncooperative patient in postoperative period may get dehydrated and pull out catheters, drains and tubes. Postoperative delirious patients are prone to fall out of bed and thus, fractures or intracranial bleeding may occur. ${ }^{[5]}$

\section{Diagnosis ${ }^{[0]}$}

Patient becomes acutely agitated, uncooperative, confused following surgery and tends to fluctuate during day. Very often, the patient is agitated during the night but calm at daytime. Main features are loss of orientation for time and impairment of attention and memory, whereas hallucinations are less frequent. However, delirium can develop at any time during hospitalization.

To assess the patient's degree of cognitive impairment, it is vital to have established a baseline for comparison. Therefore, a good preoperative evaluation should include a formal cognitive assessment in patients at risk of developing delirium. The most severe episodes of delirium usually occur in the evening (sun downing) in conjunction with excessive stimulation and disruption of the sleep-wake cycle. In addition to these criteria, patients with delirium may exhibit visual and auditory hallucinations, anxiety, agitation, altered psychomotor activity.

If delirium is suspected during postoperative period, initial focus should focus on the patient's level of consciousness by the assessments of level of orientation and any deficit in memory. Furthermore, conversation may reveal a disorganized thought process or be devoid of any content.

Delirium may be identified with the help of formal cognitive tests like, the MMSE, ${ }^{[10]}$ the confusion assessment method (CAM) score ${ }^{[11]}$ [Table 2] and the delirium writing test ${ }^{[11]}$ [Table 3]. 


\section{Table 2: Confusion assessment method*}

Feature 1: Acute onset and fluctuating course-positive responses from the family members or nurse regarding the following questions:

- Is there evidence of an acute change in mental status from the patient's baseline?

- Did the (abnormal) behavior fluctuate during the day (i.e., tend to come and go or increase and decrease in severity)?

Feature 2: Inattention- Positive response to the following questions:

- Did the patient have difficulty in focusing attention (e.g., being easily distractible or having difficulty keeping track of what was being said)?

Feature 3: Disorganized thinking- Positive response to the following question?

- Was the patient's thinking disorganized or incoherent (e.g., irrelevant conversation, illogical flow of ideas or unpredictable switching from subject to subject)?

Feature 4: Altered level of consciousness.

- Rating the patient's level of consciousness? [alert (normal), vigilant (hyperalert), lethergic (drowsy, easily aroused), stupor (difficult to arouse) or coma (unarousable)]

*The diagnosis of delirium by the confusion assessment method requires the presence of features 1 and 2 and either 3 or 4 .

\section{Table 3: Delirium writing test}

The test may be used on a preoperative day (for baseline) or whenever the patient seems mentally disturbed in the postoperative period.

1. Reluctance to write

Is the patient able to write at all (minimum his or her name)?

2. Motor impairment

Is the writing legible or is it impaired because of tremor, clumsiness, micrographia?

3. Spatial disorders

Is the patient starting on a place on the paper leaving sufficient space for the intended sentence and is he or she aligning cor rectly?

\section{Physical examination}

1. Should be focused on vital signs, fluid stai's and localizing signs of infection

2. Neurologic examination to evaluate the level of consciousness

3. History of substance abuse or withdrawal

4. Signs of trauma in all age groups

\section{Laboratory evaluation}

1. Complete blood count, for evidence of infection

2. Serum electrolytes, for evidence of hyper or hyponatremia, acidosis/ alkalosis

3. Arterial blood gas in suspicion of acid-base disturbance

4. A chest radiograph and urine analysis when pneumonia or urinary tract infections are suspected

5. ECG in the patient at risk for ischemia

\section{Risk factors ${ }^{[12-19]}$}

In all likelihood, patient predisposition, type of surgery and postoperative factors may be more important to the development of delirium than the choice of anaesthesia. A painstaking prospective study of the risk factors for postoperative delirium has been carried out. ${ }^{20]}$ Independent predictors of delirium were:

1. Age appears to place patients at greatest risk. Usually, elderly patients (more than 65 years) are on polypharmacy. However, they have decreased ability to metabolize drugs. Furthermore, visual and hearing impairments predispose elderly to disorientation. Postoperative hypoxia is mice common in the elderly given the higher incisance of cardiovascular, respiratory and ceinrovascular diseases in this group.

2. Existence of underlying brain disease (cerebrovascular accident, dementia); psychiatric illness or other medical conditions (congestive heart failure, liver disease, renal failure).

3. History of taking medicines causing altered mental status (e.g., berizodiazepines, antidepressants).

4. Patients with preexisting central nervous system disorders (dumentia, Parkinson's disease) have higher ratos of postoperative delirium.

5. Type of surgery:

a) Procedures, longer in duration place patients at increased risk of intraoperative hypoxia. ${ }^{[21]}$

b) Cardiac surgery can result in hypoperfusion and microemboli formation, causing cerebral ischaemia.

c) Orthopaedic procedures (repair of femoral neck fracture) chances of fat embolism, may cause delirium.

d) Cataract surgery is often associated with delirium due to age, loss of vision and anticholinergic sideeffects of the used ophthalmic drugs.

6. Metabolic insults include dehydration, hyponatremia, hyperglycemia, hypoglycemia, acid - base disorders, hepatic disease, renal disease and endocrine disease can cause delirium.

7. Infections like pneumonia, urinary tract infections, intra-abdominal infections and wound infections all can cause confusion in susceptible patients.

8. The sensory overload in the ICU can lead to sleep deprivation, which is a risk factor for developing delirium.

9. Postoperative pain, either uncontrolled or unaddressed has been shown to increase delirium rates. ${ }^{[7,22,23]}$

10. Abuse of alcohol/benzodiazepines, can become 
delirious secondary to withdrawal symptoms.

11. Type of anaesthesia - sometimes it is stated that delirium is less common after regional as opposed to general anaesthesia. In a large randomized controlled-study of patients undergoing elective total knee replacement, Williams - Russo et al found no statistical difference between the incidence of postoperative delirium in patients following general anaesthesia and that following epidural anaesthesia. ${ }^{[24,25]}$

\section{Differentiation from dementia}

It is important to recognize that patients with dementia are at increased risk for delirium and that delirium and dementia may coexist ${ }^{[26]}$ [Table 4].

\section{Management ${ }^{[27]}$}

Delirium is par excellence a disorder requiring a multifaceted biopsychological approach to assessment and treatment and the management strategies include both nonpharmacologic and pharmacologic interventions.

\section{Nonpharmacologic intervention Physical interventions}

Initial intervention includes general measures to support cerebral function, such as intravenous hydration and appropriate nourishment. Supplemental oxygen has been found to be highly effective in patients who developed delirium following thoracotomy for pulmonary malignancy. This intervention may also facilitate recovery in other patients whom oxygenation is not optimal, such as those with postoperative pneumonia. Physical restraints, once a mainstay in the treatment of delirium, are now used only when all pharmacologic and nonpharmacologic interventions have failed.

\begin{tabular}{lcc}
\hline $\begin{array}{l}\text { Table 4: Differentiating features of delirium and } \\
\text { dementia }\end{array}$ & \multicolumn{2}{l}{} \\
\hline Features & Delirium & Dementia \\
Onset & Acute & Insidious \\
Course & Fluctuating & Progressive \\
Duration & Days to weeks & Months to years \\
Consciousness & Altered & Clear \\
Attention & Impaired & Normal, except for \\
& severe dementia \\
Psychomotor & Increased or & Often normal \\
& changes & decreased \\
Reversibility & Usually & Rarely \\
\hline
\end{tabular}

\section{Environmental interventions}

The hospital environment is a significant factor in the management of delirium. Environmental manipulations are directed toward providing the right amount of stimulation for the patient, encouraging sleep, maximizing the patient's ability to perceive the environment accurately, maintaining safety and achieving familiarity and consistency for the patient. Ove- stimulation should be avoided, because it contributes to both confusion and insomnia. Delirious patients who are left alone without stimulation often withdraw. In such stimulations, regular interaction with hospital staff can be helpful. It is often helpful to place the delirious patient in a room close to the nursing station. Sun downing, a transient worsening of delirium that occurs in the evening hours, is presumably related, at least in part, to decreased stimulation. Hallucinations (visual and auditory) of delirium can be treated by controlled visual stimuli and by music. Adequate daytime lighting and a night light help the patient to perceive the environment accurately. Hearing aids, eyeglasses and other devices that assist sensory perception should be used whenever possible. One of the most helpful interventions is having family members stay with the patient.

\section{Cognitive interventions}

Reorientation is one of the most easily accomplished cognitive interventions. Place a clock and a calendar where the patient can see them easily. The patient should then be verbally reoriented to time and place several times over the course of the day. Repetition is recommended to compensate for memory impairment in the delirious patient.

\section{Psychological interventions}

The delusions expressed by a patient should not be directly disputed. Instead, alternative explanations of events should be offered and frequent reassurance should be given.

\section{Educational interventions}

Staff education on the recognition and treatment of delirium is essential to good delirium management. Staff members who are unfamiliar with the signs and symptoms of delirium may miss the onset of the syndrome 


\section{Pharmacologic interventions}

The intent of pharmacotherapy should be specifically to manage an unsafe or significantly disruptive behaviour that is a manifestation of the syndrome; medications are rarely effective in reversing delirium.

\section{Haloperdol}

It has become a popular choice because it has few anticholinergic side-effects, few active metabolites and can be administered orally or parenterally. Haloperidol is superior to benzodiazepines in treating delirium that has been caused by factors other than alcohol withdrawal. The dose of haloperidol is determined by the route of administration, the patient's age, the amount of agitation, the patient's risk of developing side-effects and the therapeutic setting. Low dose haloperidol ( 1 to 10 $\mathrm{mg} /$ day) improves symptoms in most patients. Haloperidol can be administered as a continuous infusion for patients who require frequent dosing.

Important side-effects of haloperidol include excessive sedation, hypotension, extrapyramidal symptoms and ventricular dysrhythmia.

\section{Benzodiazepines}

Diazepam and lorazepam are the drugs of choice in alcohol and sedative withdrawal syndromes. Extrapyramidal side effects of haloperiodol can be prevented by benzodiazepines when used as adjunct. Moreover, benzodiazepine may potentiate the effects of haloperidol, thereby allowing lower doses to be used for patients experiencing undesirable side-effects. Furthermore, haloperidol and benzodiazepine in combination can cause over sedation and life threatening hypotension and respiratory depression.

Lorazepam has several advantages owing to its sedative properties, rapid onset and short duration of action. Lower doses are necessary in elderly patients, those with hepatic diseases or those receiving compounds that undergo extensive oxidative metabolism (e.g. cimetidine and isoniazid). The recommended upper limit for intravenous lorazepam is $2 \mathrm{mg}$ every four hours. Giving adequate initial doses reduce the risk of paradoxical excitement.

The major advantage of benzoidazepines is that, their effects can be rapidly reversed with flumazenil.

A treatment regimen for severe cases requiring prompt, aggressive control of symptoms is outlined below:[28]

- Administer 1-10 mg haloperidol (intramuscularly or intravenously) depending on level of disturbance and likely tolerance (having considered age, physical status and risk of side effects).

- Observe patient for 20-30 minutes. If the patient remains unmanageable but has not had any adverse effects, double the dose and continue monitoring.

Repeat the cycle until an acceptable response occurs or side effects occur.

- Up to $2 \mathrm{mg}$ of lorazepam may be administered intravenously or intramuscularly every four hours and may be beneficial in allowing a lower dose of antipsychotics to be used in cases in which extrapyramidal side effects occur.

Monitor respiratory functions and level of sedation carefully. Consider administering fluma-zenil if there is evidence of significant toxicity.

\section{Other pharmacological interventions ${ }^{[29]}$}

Following drugs can be used in special circumstances. Opioid: For patients with terminal disease and in those whom pain is an aggravating factor, opioids may provide best palliation.

Vitamin B: When vitamin B deficiencies are considered a possible cause, replacement of deficient vitamins is necessary, especially in alcoholics. ${ }^{[30]}$

Physostigmine: It is effective in delirium caused by anticholinergic syndrome. ${ }^{[30]}$

Note: Inspite of all the inteventions mentioned above, if delirium progresses to coma, standard treatment for control of airway, breathing and circulation should be instituted.

\section{Summary / Conclusion}

Fifteen dddpercent of the older patients have delirium at the time of admission to the hospital and another 25\% develop the problem during the postoperative period. Patients with underlying dementia are at higher risk of having delirium than those with intact cognition. In addition, dehydration, multiple medications, infections, urinary retention and severe underlying illness may all contribute to the development of delirium. Instruments have been developed that are not only sensitive in diagnosing delirium but also differentiate it from dementia with the delirium rating scale $e^{[31]}$ and the confusion 
assessment method. ${ }^{[32]}$ In general, any change in mental status over baseline should be ascribed to delirium until proven otherwise and a diligent search for its cause pursued because delirium is associated with increased mortality.

\section{References}

1. Folstein MF, Folstein SE, McHugh PR. "Mini - Mental State". A practical method for grading the cognitive state of patients for the children. J Psychiatr Res 1975;12:189-98.

2. Cole MG, Primeau FJ. Prognosis of delirium in elderly hospital patients. Can Med Assoc J 1993;149:41-6.

3. Gustafson Y, Berggren D, Branstorm B, Bucht G, Norberg A, Hansson LI, et al. Acute confusional states in elderly patients treated for femoral neck fracture. J Am Geriatr Soc 1988;36:525-30.

4. Chung F. Postoperative mental dysfunction. In: McLesky $\mathrm{CH}$, editor. Geriatric Anesthesiology. Williams and Wilkins: 1997. p. 487-95.

5. Mcintosh TK, Bush HL, Yeston NS, Grasberger R, Palter M, Aun $\mathrm{F}$, et al. Beta-endorphin, cortisol and postoperative delirium: $\mathrm{A}$ preliminary report. Psychoneuroendocrinology 1985;10:303-13.

6. Jacquiline ML, Laura PS, Elisabeth A, Mullen BS, Yun W, Linnea V. Preoperative depression increases the risk of postoperative delirium in geriatric patients. Anesthesiology 2004;101:A66.

7. Linnea EV, Laurea PS, Yun W. The role of pain and medication on postoperative delirium. Anesthesiology 2004;101:A39.

8. Gustafson Y. Postoperative delirium - a challenge for the orthopedic team. Acta Orthop Scand 2004;75:375-7.

9. Winawar N. Postoperative Delirium. Med Clin North Am 2001;85:1229-39.

10. Dyer CB, Ashton CM, Teasdale TA. Postoperative delirium. A review of 80 primary data-collection studies. Arch Intern Med 1995;155:461-5.

11. Aakerlund LP, Rosenberg J. Writing disturbances: An indicator for postoperative delirium. Int J Psychiatr Med 1994;24:245-57.

12. Leung JM, Sands LP, Mullen EA, Wang Y, Vaurio L. Are preoperative depressive symptoms associated with postoperative delirium in geriatric patients? J Geriontol 2005;60:1563-8.

13. Kawaguchi $Y$, Kanamori M, Ishihara $H$, Abe $Y$, Nobukiyo M, Sigeta $\mathrm{T}$, et al. Postoperative delirium in spine surgery. Spine $\mathrm{J}$ 2006;6:164-9.

14. Leung JM, Sands LP, Vaurio LE, Wang Y. Nitrous oxide does not change the incidence of postoperative delirium or cognitive decline in elderly surgical patients. Br J Anaesth 2006;96:754-60.

15. Sharma PT, Zakriya K, Pauldine RW, Mears SC, Sieber F. Emergence delirium predicts postoperative delirium following hip fracture repair. Anesthesiology 2004;101:A67.

16. Sieber FE, Pauldine R. Anaesthesia for the elderly. In: Miller RD, editor. Miller's Anaesthesia. $6^{\text {th }}$ ed. Elsevier Churchill Livingstone: Philadelphia; 2005. p. 2441-2.
17. Mecca RS. Postoperative recovery. In: Barash PG, Cullen BF, Stoelting RK, editors. Clinical Anaesthesia. $5^{\text {th }}$ ed. Lippincott Williams and Wilkins: Philadelphia; 2006. p. 1402.

18. Muravchick S. Anaesthesia for the elderly. In: Healy TE, Knight PR, editors. Wylie and Churchill Davidsons. A Practice of Anaesthesia. $7^{\text {th }}$ ed. Arnold: London; 2003. p. 981-93.

19. Solomon D, Solomon DE, Tomasovic JJ. Neurologic Consultation. In: Clinical Anaesthesia Practice. $2^{\text {nd }}$ ed. Kirby RR, Gravenstein N, Lobato EB, Graven-stein JS, editors. WB Saunders Company: Philadelphia; 2002. p.188-9.

20. Marcantonio ER, Goldman L, Mangione CM, Ludwig LE, Muraca $\mathrm{B}$, Haslauer CM, et al. A clinical prediction rule for delirium after elective noncardiac surgery. JAMA 1994;271:134-9.

21. Lepouse C, Lautner CA, Liu L, Gomis P, Leon A. Emergence delirium in adults in the post-anaesthesia care unit. $\mathrm{Br}$ j Anaesth 2006;96:747-53.

22. Lynch EP, Lazor MA, Gellis JE, Orav J, Goldman L, Marcantonio ER. The impact of postoperative pain on the development of postoperative delirium. Int Anesth Res Soc 1998;86:781-5.

23. Vaurio LE, Sands LP, Wang Y, Mullen EA, Leung JM. Postoperative delirium: The importance of pain and pain management. Anesth Analg 2006;102:1267-73.

24. Williams-Russo P, Sharrock NE, Mattis S, Szatrowski TP, Charlson ME. Cognitive effects after epidural vs general anaesthesia in older adults: A randomized trial. JAMA 1995;274:44-50.

25. Bryson GL, Wyand A. Evidence - based clinical update: General anaesthesia and the risk of delirium and pos-operative cognitive dysfunction. Can J Anaesth 2006;53:669-77.

26. Tune L, Ross C. Delirium. In: Coffy CE, Cummings JL, editors. Textbook of geriatic neuropsychiatry. American Psychiatric Press: Washington, DC; 1994. p. $351-68$.

27. Bitsch M, Foss N, Kristensen B, Kehlet H. Pathogenesis of and management strategies for postoperative delirium after hip fracture: A review. Acta Orthop Scand 2004;75:378-89.

28. Meagher DJ. Delirium: Optimizing management. BMJ 2001;322:144-9.

29. Deilirium PP. In: Tallis RS, Fillit HM, editor. Brocklehurst's textbook of Geriatic Medicine and Gerontology. $6^{\text {th }}$ ed. Churchill Livingstone: London; 2003. p. 770-1.

30. Cavaliere F, D'Ambrosio F, Volpe C, Masieri S. Postoperative delirium. Curr Drug Targets 2005;6:807-14.

31. Trzepacz PT, Baker RW, Greenhouse J. A symptom rating scale for delirium. Psychiatr Res 1988;23:89-97.

32. Inouye SK, van Dyck CH, Alessi CA, Balkin S, Siegal AP, Horwitz RI. Clarifying confusion. The confusion assessment method: A new method for detection of delirium. Ann Intern Med 1990;113:941-8.

Source of Support: Nil, Conflict of Interest: None declared. 